\title{
Ignition Regimes in Rapid Compression Machines
}

\author{
Kevin P. Grogan ${ }^{*, a}$, S. Scott Goldsborough ${ }^{\mathrm{b}}$, Matthias Ihme ${ }^{\mathrm{a}}$ \\ ${ }^{a}$ Department of Mechanical Engineering, Stanford University, Stanford, CA 94305 \\ ${ }^{b}$ Center for Transportation Research, Argonne National Laboratory, 9700 S. Cass Avenue, Argonne, IL 60439
}

\begin{abstract}
A rapid compression machine is an experimental apparatus used to study ignition chemistry at conditions that are relevant to internal combustion engines and gas turbines. However, due to the operating characteristics of these devices, heat transfer effects and inhomogeneous ignition events can be encountered. Hence, this paper develops a combustion regime diagram, which incorporates these effects in order to better understand these physical influences on the measurements. This diagram employs familiar Damköhler number and Reynolds number scaling, and seeks to provide an operational guide for rapid compression machine experiments. This diagram is compared to experimental data and good agreement is found.
\end{abstract}

Key words: Rapid compression machine, Regime diagram, Ignition, Heat transfer, Turbulence

\section{Nomenclature}

Upper-case Roman Letters

$\mathcal{A} \quad$ Arrhenius factor

AR Aspect ratio

$B \quad$ Ignition delay exponential prefactor

C Constant

CR Compression ratio

$\mathrm{Da}_{\mathrm{t}} \quad$ Turbulent Damköhler number

$E_{\mathrm{A}} \quad$ Overall activation energy

$\Delta H_{\mathrm{C}} \quad$ Heat of combustion

$L \quad$ Stroke length

M Mach number

$N_{\mathrm{s}} \quad$ Number of species

Pr Prandtl number

$\mathcal{R} \quad$ Universal gas constant

$R_{t} \quad$ Turbulent Reynolds number

$S_{\mathrm{L}} \quad$ Laminar flame speed

$T \quad$ Temperature

$\mathcal{T} \quad$ Intensity

$U_{\mathrm{P}} \quad$ Piston velocity

Lower-case Roman Letters

a Speed of sound

$b \quad$ Characteristic heat transfer length scale

c Specific heat

d Diameter

$e \quad$ Internal energy

$g \quad$ Kinematic viscosity scaling constant

$k \quad$ Turbulent kinetic energy

$l \quad$ Integral length scale

$n \quad$ Turbulence decay exponent

$p \quad$ Pressure

$t \quad$ Time

$u \quad$ Velocity

\footnotetext{
* Corresponding author.

Email addresses: kgrogan@stanford.edu (Kevin P. Grogan), scott.goldsborough@anl.gov (S. Scott Goldsborough), mihme@stanford.edu (Matthias Ihme)
}

Preprint submitted to Elsevier 


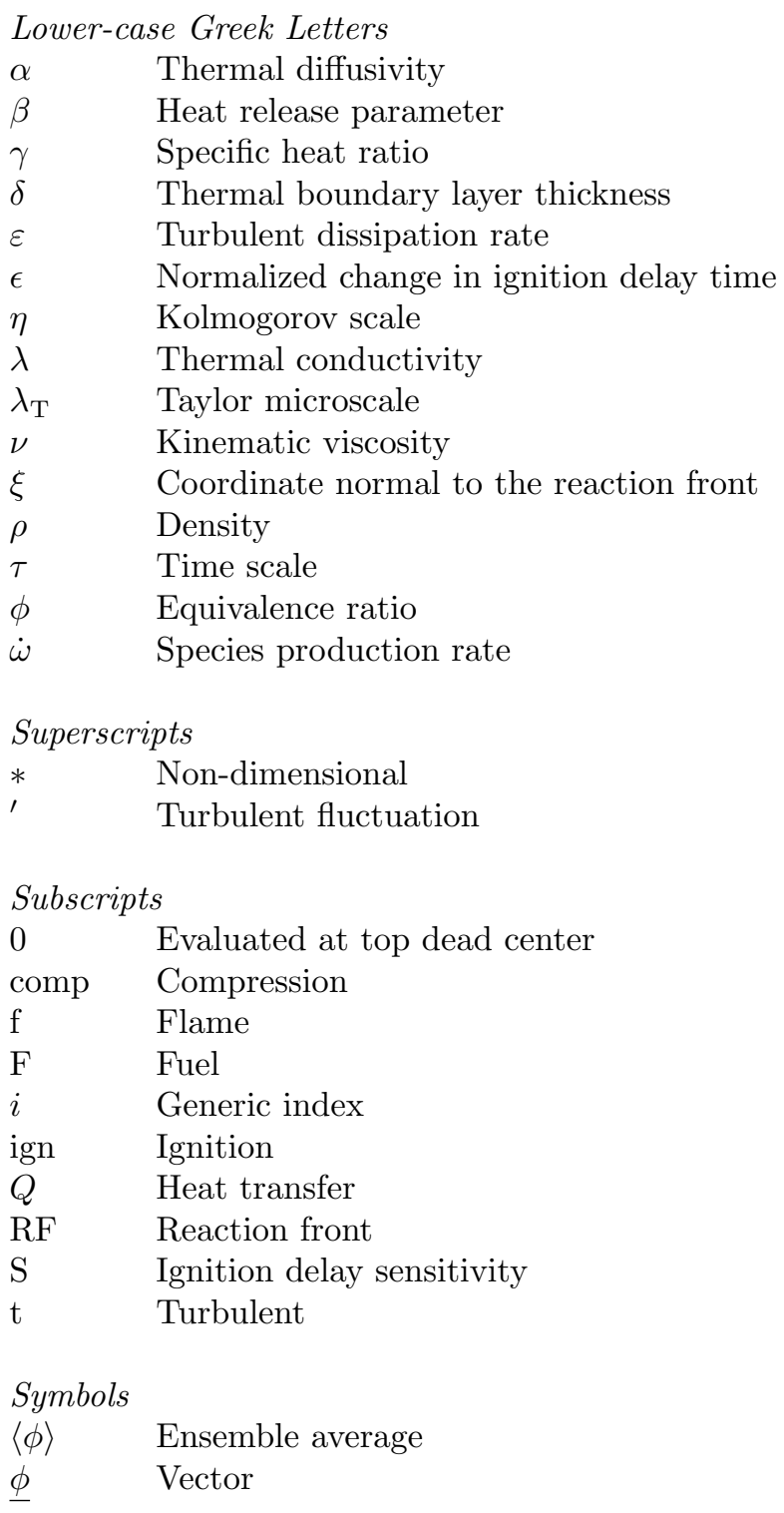

\section{1. Introduction}

Rapid compression machines (RCMs) [1], like shock-tubes [2], and flow/jet reactors [3], are used to study fuel decomposition and ignition behavior at elevated temperature and pressure conditions that are representative of combustion engine environments. These different experimental devices are designed to isolate chemical kinetic phenomena from other complex processes that occur within the combustion chamber during engine operation such as spray breakup, fuel evaporation, and mixing. There is a long history of development of RCMs, and modern configurations are generally well-characterized over a range of conditions. Typical temperature and ignition delay ranges accessed by RCMs are depicted in Fig. 1, where it can be seen that regions of overlap exist between the different types of chemical reactors.

RCMs typically explore ignition behavior using realistic fuel loadings $\left(\phi \approx 0.5-2, \mathrm{O}_{2} \approx 5-21 \%\right)$, which is similar to what is employed in operating engines. Other devices, such as shock-tubes, are usually operated under lean or dilute conditions $\left(\phi<0.5, \mathrm{O}_{2}<1 \%\right)$ in order to minimize thermal feedback and inhomogeneities that can result from exothermic and endothermic processes, as well as other operational challenges such as excessive pressure rise rates. Complementary data can be acquired through the use of a variety of apparatuses towards the development of predictive reaction mechanisms and other combustion models.

Idealized reactors like RCMs can be influenced by physical processes which result in coupled chemico-physical phenomena, and this leads to non-ideal experimental conditions. Measurements and interpretation of datasets under these scenarios can be complicated. In RCMs such processes can include heat loss and complex fluid dynamics, as well as turbulent fluctuations and the development of non-uniform or deflagrative ignition.

Heat loss is particularly notable in RCMs since piston compression and test times can be long (e.g., $\tau_{\text {comp }}=15-$ $80 \mathrm{~ms}, \tau_{\text {ign }}=10-100 \mathrm{~ms}$ ), while the walls of the reaction chamber are substantially cooler than the reacting gas. 
During the test period, a boundary layer forms and diffuses into the test section such that a significant variation of temperature can be present throughout the RCM, and no definable core exists at very long test times. Additionally, at some point the reactivity can be quenched, and ignition does not occur.

Complex fluid dynamics can exist within RCMs, and their influence on ignition has been recognized for many years. A particular focus of late has been the generation of a toroidal vortex in the reaction chamber during piston compression, where this can occur even for mixtures that are initially quiescent [4]. The fluid dynamic structure that develops at the interface between the piston and the cylinder wall can evolve during the test period and mix the "adiabatic core" gas with cooler boundary layer gases leading to highly non-homogeneous conditions within the reaction chamber.

Park and Keck [4] first suggested the use of a crevice volume machined around the circumference of the piston to capture the boundary layer during compression and prevent the formation of this vortex. Lee and Hochgreb [5] expanded upon this idea and conducted computational fluid dynamics (CFD) simulations of the process to devise guidelines for an adequate crevice configuration.

Mittal and Sung [6] used CFD simulations and conducted experimental measurements with acetone planar laser induced fluorescence (PLIF) to better understand the evolution of the fluid dynamic motion within the reaction chamber and verify the performance of their crevice design. Their measurements confirmed the capability of the crevice to suppress the roll-up vortex and achieve very uniform conditions across the "adiabatic core" for high pressure cases studied ( $p=40$ bar). Normalized root mean squared (RMS) temperatures under these conditions were near $T^{\prime} /\langle T\rangle=0.01$, which is within the experimental uncertainties of the PLIF measurements, while average gradients were on the order of $\mathrm{d} T / \mathrm{d} \xi=2 \mathrm{~K} / \mathrm{mm}$, with peak values near $5-10 \mathrm{~K} / \mathrm{mm}$. However, for the low pressure tests ( $p=12$ bar), the vortex formation was not suppressed due to the three times larger thermal diffusivity of the gas, and normalized RMS temperatures increased to $T^{\prime} /\langle T\rangle=0.02$ with mean gradients closer to $\mathrm{d} T / \mathrm{d} \xi=4 \mathrm{~K} / \mathrm{mm}$ and peak values near $10-15 \mathrm{~K} / \mathrm{mm}$.

Turbulent fluctuations within the reaction chamber can generate or amplify inhomogeneities within the "adiabatic core" during piston compression, and interact with the chemical kinetic processes during the delay period including the main heat release process. The development of turbulent conditions however has not been investigated widely, especially outside of studies where bulk fluid motion is not suppressed (e.g., via piston crevices). Turbulence can be introduced into the RCM via a variety of means including latent turbulence from the filling process, turbulence generated by boundary layer instabilities, development of corner vortices during the compression stroke, and production of turbulence from the mean strain induced by the compression $[7,8]$.

Guibert et al. [9] studied the decay of the mean total kinetic energy during the delay period using particle image velocimetry (PIV) techniques. They noted that under short ignition delay times, the turbulence can interact with the chemical heat release. The configuration used for their investigation employed a mesh at the interface between the reaction chamber and compression cylinder to generate turbulent flow fields. As such, it is difficult to directly apply their findings to typical RCM configurations where the bulk flow field is suppressed.

Ihme [7] developed a stochastic multi-scale model to investigate the evolution of velocity and temperature fluctuations during piston compression and the ignition delay period, covering a range of geometric and operating conditions. This model indicated that increased compression ratios can significantly enhance local stratification, while the heat release profile and ignition timing can be substantially altered under low Damköhler conditions.

Deflagrative ignition, which is referred to as "mild ignition" in this work, occurs when flames develop within the reaction chamber. These develop from localized ignition kernels and can consume the entire mixture, or promote volumetric ignition of the remaining unburned charge. Ignition delay times are generally shortened during mild ignition events, while the rates of pressure rise are much slower than under uniform ignition conditions. The term "weak ignition" is not used here to describe these events, as this terminology has been used in past works to identify regimes where autoignition is not fully chemically branched, and is significantly influenced by chemical energy release, evolving from a relatively slow process and transitioning to rapid heat release at the point of main ignition.

Mild ignition phenomena were first documented in an RCM by Livengood and Leary [10] who employed Schlieren techniques and high speed photography using an optically accessible configuration. They identified regions of thermal stratification along with the evolution of flame fronts during autoignition. By considering different fuels, they concluded that the stratification was primarily caused by the piston motion, where the piston scrapes the boundary layer from the cylinder walls, producing a turbulent region at the periphery of the chamber.

Many researchers, including Oppenheim and co-workers [11, 12], Adomeit and co-workers [13, 14], Yamashita et al. [15] and Uygun et al. [16], subsequently investigated mild ignition processes within shock-tubes using Schlieren techniques. These groups studied influences of thermal and reactivity gradients generated by their configurations, and they explored the sensitivities of various fuels to these gradients.

Wooldridge and coworkers $[17,18,19,20]$ studied mild ignition phenomena in syngas and iso-octane mixtures using a RCM where a wide range of fuel loadings $(\phi=0.1-0.7$ and $\phi=0.2-1$, respectively) and pressures $(p=3-20$ bar and $5-20$ bar, respectively) were covered in the intermediate temperature regime $(T=800-1100$ $\mathrm{K}$ and $T=900-1050 \mathrm{~K}$, respectively). They employed high speed chemiluminescence photography to distinguish between mild and uniform ignition events, and determined ignition front velocities for some of their non-uniform ignition data. They also developed sensitivity diagrams for some of their mixtures in order to identify thermodynamic 
regimes where mild ignition can be observed. An assumed thermal gradient near $\mathrm{d} T / \mathrm{d} \xi=5-10 \mathrm{~K} / \mathrm{mm}$ was employed to help delineate the different regions of ignition.

Strozzi et al. [21] investigated mild ignition of methane/air mixtures using a square cross-section, flat piston configuration. They employed toluene PLIF measurements to characterize thermal inhomogeneities within the reaction chamber, and PIV to ascertain the characteristics of the velocity fields. Their measurements showed regions of high RMS temperature and thermal gradients $\left(T^{\prime} /\langle T\rangle>0.07\right.$ and $\left.\mathrm{d} T / \mathrm{d} \xi>100 \mathrm{~K} / \mathrm{mm}\right)$. The data were used to explore differences between spontaneous ignition front propagation and deflagration within the mixtures, and found that under the conditions examined, most of the heat release occurred via heterogeneous ignition.

Figure 1 is representative of the test conditions explored by three categories of chemical reactors; however, it is an incomplete description of the capabilities of a given facility. A more thorough description incorporates the relevant physical time scales of the system; hence, it is the ambition of this paper to elucidate these physical processes for rapid compression machines as was done previously for flow reactors [22]. Specifically, by employing essential scaling analysis of processes involving hydrodynamics, turbulence, heat transfer, ignition, and combustion, a regime diagram is developed to delineate relevant ignition regimes in RCMs. The utilization of this regime diagram can lead to better experimental designs as well as operating protocol, which can more confidently target ignition regimes of interest. For instance, studies focused on chemical kinetics of fuel decomposition should be conducted in a regime where thermal quenching will not occur and ignition is absent of deflagrative and detonative processes.

The remainder of this manuscript is organized as follows: a description of the various physical phenomena affecting ignition is provided in the next section, and scaling relationships distinguishing different ignition regimes are derived; these derivations are followed by a representative comparison to experimental data available in the literature and discussion.

\section{Ignition regime diagram for rapid compression machines}

RCMs typically seek to operate in regimes with simple gas dynamics for chemical kinetic studies. However, the action of compressing the test gas induces perturbations to the flow field, which can have a significant effect on the character of the ignition event. Additionally, heat loss to the walls complicates the interpretation of RCM measurements and can lead to thermal quenching.

To decipher the governing parameters within an RCM at the test conditions, consider the energy equation:

$$
\rho c_{v} \frac{\partial T}{\partial t}=-\rho c_{v} \underline{u} \cdot \nabla T-\sum_{i=1}^{N_{\mathrm{s}}} e_{i} \dot{\omega}_{i}+\nabla \cdot(\lambda \nabla T),
$$

where work and mass diffusion terms are ignored. Work terms are considered negligible outside of the compression stage, and inhomogeneities in species concentration are likely small since the boundaries are non-catalytic. Nondimensionalizing Eq. (1) with respect to the condition at the end of compression or top dead center (TDC), the energy equation becomes

$$
\frac{\partial T^{*}}{\partial t^{*}}=-\underline{u}^{*} \cdot \nabla^{*} T^{*}-\frac{\gamma \mathrm{Da} \mathrm{a}_{\mathrm{t}}}{\mathcal{A}} \sum_{i=1}^{N_{\mathrm{s}}} e_{i}^{*} \dot{\omega}_{i}^{*}+\frac{\gamma}{\operatorname{Pr} \mathrm{Re}_{\mathrm{t}}} \nabla^{*} \cdot\left(\lambda^{*} \nabla^{*} T^{*}\right),
$$

where the superscript "*” indicates a non-dimensionalized variable, Dat is the turbulent Damköhler number, $\gamma$ is the specific heat ratio, and $\mathcal{A}$ is the Arrhenius factor. The Arrhenius factor is discussed more extensively in Sec. 2.2.1. The Prandtl number is defined as $\operatorname{Pr}=\nu / \alpha$, and $\operatorname{Re}_{t}$ is the turbulent Reynolds number. The turbulent Damköhler number is defined as [7]:

$$
\mathrm{Da}_{\mathrm{t}}=\frac{\tau_{\mathrm{t}}}{\tau_{\mathrm{ign}}}
$$

where $\tau_{\mathrm{t}}$ is the turbulent time scale defined as the ratio of the integral length scale to the turbulent velocity fluctuation $\left(\tau_{\mathrm{t}}=l / u^{\prime}\right)$, and $\tau_{\mathrm{ign}}$ is the ignition delay time for the mixture. The ignition delay is the chemical kinetics time and is considered to be unaffected by turbulence and heat transfer in this definition. Additionally, this paper defines all relevant variables in algebraic expressions with respect to the conditions at TDC unless otherwise noted. Hence, the turbulent Damköhler number compares the turbulent mixing time scale to the chemical time scale of the mixture.

The turbulent Reynolds number is defined as

$$
\mathrm{Re}_{\mathrm{t}}=\frac{u^{\prime} l}{\nu},
$$

where $\nu$ is the kinematic viscosity. The turbulent Reynolds number can be interpreted as a competition between a viscous dissipation time scale and a turbulent mixing time scale:

$$
\operatorname{Re}_{\mathrm{t}}=\frac{\tau_{\nu}}{\tau_{\mathrm{t}}}
$$


where the viscous time scale is defined as $\tau_{\nu}=l^{2} / \nu$. Equation (2) assumes that the heat transfer time scale is linearly related to the viscous time scale through the Prandtl number: $\tau_{Q}=\operatorname{Pr} \tau_{\nu}$.

The non-dimensional energy equation governing the ignition process given by Eq. (2) is shown to be parameterized by the turbulent Damköhler number and the turbulent Reynolds number. Considering both parameters, a combustion regime diagram for RCMs was developed and is presented in Fig. 2. The subsequent sections derive the demarcations shown in Fig. 2 starting with the demarcations pertaining to the turbulence levels in Sec. 2.1, then the demarcations associated with the characterization of the ignition events (i.e., strong, mixed, mild, or pre-ignition) in Sec. 2.2, and finally, the demarcations primarily associated with heat transfer effects in Sec. 2.3.

\subsection{Turbulence}

Turbulence can have a substantial effect on the quality of ignition and can lead to erroneous assessments or predictions of ignition delay. Turbulence is a highly nonlinear and dimensional process which cannot be easily incorporated into models of RCMs with high accuracy. Hence, regimes in which turbulence substantially affects the ignition process in a chemical kinetic experiment should be avoided.

A schematic of the turbulent flow field at TDC is shown in Fig. 3. The turbulent flow field in an RCM consists of a diversity of length scales including the integral length scale $l$, the Taylor microscale $\lambda_{\mathrm{T}}$, and the Kolmogorov length scale $\eta$. Ignition kernel formation occurs at an intermediate scale between the integral length scale and the Kolmogorov scale, and Peters et al. [23] showed through dissipation element theory that kernel formation occurs at length scales similar to the Taylor microscale. Additionally, a boundary layer of cold fluid, with length scale $\delta$, develops at the wall of an RCM. As discussed in the Introduction, the dynamics of this boundary layer has a significant effect on the temperature field within the RCM.

\subsubsection{Quiescent ignition}

When studying chemical kinetics, a quiescent flow would be desirable for RCM experiments. Quiescent ignition occurs when the reaction proceeds uniformly within the test section, and there is no turbulence in the flow. When the flow is quiescent and ignition is not quenched due to heat loss to the walls, the mixture ignites uniformly. As demonstrated by Eq. (5), the turbulent Reynolds number is the ratio of the viscous and turbulent time scales. Hence, for short viscous time scales compared to the turbulent mixing time scale, the mixture homogenizes throughout the test section leading to a uniform ignition event. Therefore, the quiescent region is demarcated by the line $\mathrm{Re}_{\mathrm{t}}=1$. This scaling is included as a vertical line bounding the quiescent regime in Fig. 2. However, the quiescent regime is rather difficult to achieve experimentally without other non-idealities dominating; the piston velocity would be such that appreciable radical and boundary layer build-up would occur during the compression stroke. Additionally, homogeneous ignition can also occur when the mixture is turbulent, and the delineation for this regime is examined in a Sec. 2.2.1.

\subsubsection{Turbulence decay}

For long ignition delay times, the turbulence within an RCM may decay to a level where it has no effect on the quality of the ignition. In these cases, the core fluid ignites uniformly without a flame or detonation front forming. Assuming homogeneous turbulence at the end of the compression stroke, the decay of the turbulence can be modeled with the following power laws [24]:

$$
\begin{aligned}
& \frac{k_{\text {ign }}}{k}=\left(1+\frac{\tau_{\text {ign }}}{\tau_{\mathrm{t}}}\right)^{-n}, \\
& \frac{\varepsilon_{\text {ign }}}{\varepsilon}=\left(1+\frac{\tau_{\text {ign }}}{\tau_{\mathrm{t}}}\right)^{-(1+n)},
\end{aligned}
$$

where $k$ is the turbulent kinetic energy, $\varepsilon$ is the turbulent dissipation rate, the subscript "ign" indicates the state at the time of ignition, and the exponent $n$ is taken to be 1.3 from Pope [24]. By reformulating the turbulent and viscous time scales in terms of $k$ and $\varepsilon$ using dimensional analysis, the turbulent Reynolds number given in Eq. (4) can be rewritten as

$$
\operatorname{Re}_{\mathrm{t}}=\frac{k^{2}}{\varepsilon \nu} .
$$

Dividing Eq. (6b) by the square of Eq. (6a), assuming that viscosity does not vary appreciably during the induction period, and substituting the turbulent Reynolds number given in Eq. (7), Eqs. (6) can be expressed as

$$
\frac{\mathrm{Re}_{\mathrm{t}}}{\mathrm{Re}_{\mathrm{t}, \text { ign }}}=\left(1+\frac{\tau_{\text {ign }}}{\tau_{\mathrm{t}}}\right)^{n-1} .
$$

The flow becomes quiescent at the time of ignition when $\operatorname{Re}_{t, i g n}=1$. Applying this criterion, substituting the definition of the turbulent Damköhler number, and rearranging, Eq. (8) becomes

$$
\mathrm{Da}_{\mathrm{t}}=\frac{\mathrm{Re}_{\mathrm{t}}^{-m}}{1-\mathrm{Re}_{\mathrm{t}}^{-m}},
$$


where $m=(n-1)^{-1} \approx 3.3$. For $\operatorname{Re}_{\mathrm{t}} \gg 1$, Eq. (9) reduces to the following:

$$
\mathrm{Da}_{\mathrm{t}}=\mathrm{Re}_{\mathrm{t}}^{-m} .
$$

If the Damköhler number is below the demarcation given in Eq. (10), the flow becomes quiescent before the time of ignition, and therefore, the core fluid is likely to ignite uniformly. The delineation given by Eq. (10), is shown in Fig. 2 as having a slope of -3.3 .

\subsubsection{Estimation of turbulence statistics}

Since turbulence statistics are rarely measured in RCMs, the turbulent time scale can be estimated assuming the following relations:

$$
\begin{aligned}
\mathcal{T}_{u} & =\frac{u^{\prime}}{U_{\mathrm{P}}}, \\
\mathrm{C}_{l} & =\frac{l}{d},
\end{aligned}
$$

where $\mathcal{T}_{u}$ is the turbulence intensity, and $U_{\mathrm{p}}$ is the characteristic velocity of the piston in the RCM (either known or estimated by stroke length and compression time), $\mathrm{C}_{l}$ is a proportionality constant, and $d$ is the diameter of the RCM. The turbulence intensity is used to relate the piston velocity to the RMS velocity. This quantity is most likely machine-dependent and can be affected by other factors including crevice, piston, compression chamber, and reaction-chamber design. However, the ratio of the integral length scale to the diameter is unlikely to vary appreciably between machines.

As discussed in the introduction, much work has been undertaken to minimize the hydrodynamic fluctuations through the insertion of optimized crevices in $\mathrm{RCMs}[4,5,6]$. The present analysis incorporates the effects of geometric complexity of crevice design through the turbulent intensity, $\mathcal{T}_{u}$. For highest accuracy, this is a quantity that would need to be measured for a particular RCM.

Using Eqs. (11), the turbulent time scale can be estimated as:

$$
\tau_{\mathrm{t}}=\left(\frac{\mathrm{C}_{l}}{\mathcal{T}_{u}}\right) \frac{d}{U_{\mathrm{p}}} .
$$

Hence, the turbulent time scale is dependent on the geometry of the RCM as indicated by $\mathcal{T}_{u}$ and $d$, and the speed of the piston $U_{\mathrm{P}}$.

\subsection{Chemistry}

Manifestly, the chemistry of the test gas is of great importance in all regimes of chemical kinetic experiments. However, within regimes that delineate the quality of ignition, the chemical sensitivities of the test mixture dominate. Mild ignition is a chemico-physical phenomenon driven by turbulence and chemical sensitivity. Mild ignition differs from strong ignition phenomena due the inhomogeneous nature of the ignition event, which is characterized by ignition kernel formation. The ignition kernels form from turbulent fluctuations in temperatures in the test gas. From the presumption of a turbulent flow field, several regime demarcations can be postulated to segregate homogeneous and mild ignition regimes in RCMs.

First, the regime demarcation for the strong ignition will be discussed, and subsequently, the delineation for the mild ignition regime will be examined.

\subsubsection{SWACER and the strong ignition limit}

Shock Wave Amplification by Coherent Energy Release (SWACER) [25, 26] is a mechanism that explains the transition of isolated ignition kernels to detonation. SWACER theory predicts a spontaneous transition into a detonation wave if there is a coherent coupling of pressure waves and heat release at the ignition kernel [27]. The transition criterion is given as

$$
\frac{a}{u_{\mathrm{RF}}}=1
$$
detonation wave in one-dimensional simulations and found acceptable agreement between Eq. (14) and the computed transition. The SWACER mechanism has been found to be relevant in inhomogeneous ignition phenomena [30]. Additional mechanisms for deflagration-to-detonation transition (DDT), including kernel-wall-interaction and kernelkernel-interaction, are discussed by Blumenthal et al. [31] in the context of shock-tube systems. 
Assuming an Arrhenius form, the ignition delay is given by

$$
\tau_{\text {ign }}=B \exp \left(\frac{E_{\mathrm{A}}}{\mathcal{R} T}\right),
$$

where $B$ is an exponential prefactor that is generally a function of density, $E_{\mathrm{A}}$ is the activation energy, and $\mathcal{R}$ is the universal gas constant. Taking the partial derivative of Eq. (15) with respect to temperature and defining the Arrhenius factor as $\mathcal{A}=E_{\mathrm{A}} / \mathcal{R} T$, the partial derivative of the ignition delay is given as

$$
\frac{\partial \tau_{\text {ign }}}{\partial T}=-\frac{\mathcal{A}}{T} \tau_{\text {ign }}
$$

Following Peters et al. [23], the Taylor microscale, $\lambda_{\mathrm{T}}$, is employed as the characteristic dimension of a flame kernel. From this, the temperature gradient is approximated as

$$
\frac{\partial T}{\partial \xi} \approx-\frac{T^{\prime}}{\lambda_{\mathrm{T}}}=-\mathcal{T}_{T} \frac{T}{\lambda_{\mathrm{T}}}
$$

where $\mathcal{T}_{T}$ is the temperature fluctuation level. Substituting Eqs. (16) and (17) into Eq. (14) the following relation is obtained:

$$
a \mathcal{T}_{T} \tau_{\text {ign }} \frac{\mathcal{A}}{\lambda_{\mathrm{T}}}=1
$$

Using homogeneous turbulence theory [24], the Taylor microscale is given by

$$
\lambda_{\mathrm{T}}=\sqrt{10 \nu \tau_{\mathrm{t}}} .
$$

Defining the relevant Mach number as

$$
\mathrm{M}=\frac{U_{\mathrm{p}}}{a},
$$

and substituting the definitions of the turbulent Reynolds number and the turbulent Damköhler number, given in Eqs. (4) and (3), respectively, the SWACER criterion is found to be

$$
\operatorname{Da}_{\mathrm{t}}=\left(\frac{\mathcal{T}_{T}}{\mathcal{T}_{u}}\right) \frac{\mathcal{A}}{\mathrm{M} \sqrt{10}} \operatorname{Re}_{\mathrm{t}}^{1 / 2}
$$

This equation shows the influence of compressibility and chemistry by the appearance of the Mach number and the Arrhenius factor, respectively; however, independence of the SWACER criterion to the design of the RCM is shown since it is likely that $\mathcal{T}_{T} \propto \mathcal{T}_{u}$. This criterion designates the propensity of a mixture to ignite either as a detonation or as a supersonic autoignition wave. The latter is interpreted in the context of an RCM as a strong ignition event. The demarcation between mixed and strong ignition, given by Eq. (21), is shown in Fig. 2 and has a slope of $1 / 2$.

\subsubsection{Sankaran criterion}

Sankaran et al. [32], by extending the work of Gu et al. [29], postulated that a comparison between the velocity of the reaction front of an ignition kernel to the laminar flame speed yields a demarcation that indicates the propensity of a mixture towards mild ignition. This criterion is given as

$$
\frac{S_{\mathrm{L}}}{u_{\mathrm{RF}}}=1
$$

where $S_{\mathrm{L}}$ is the laminar flame speed, and $u_{\mathrm{RF}}$ is the speed of the ignition front. The Sankaran criterion is qualitatively similar to the SWACER criterion. However, the Sankaran criterion is meant to demarcate the regime in which the mixture forms flame kernels, and the SWACER criterion is meant to indicate the propensity of a flame kernel to transition to a detonation. Hence, the region bounded by these criteria is a transitional regime where a flame kernel readily transitions into a detonation wave or experiences mixed ignition. A mixed ignition event is characterized as a transient ignition state where flame fronts are produced before the mixture undergoes uniform ignition, as discussed in the Introduction.

The evaluation of the speed of the reaction front is the same as that for the SWACER criterion. Using a two-zone model for a laminar premixed flame [33], the laminar flame speed is approximated as

$$
S_{\mathrm{L}}^{2} \approx 2 \alpha \frac{\dot{\omega}_{\mathrm{F}}\left(T_{\mathrm{f}}\right)}{\rho_{\mathrm{F}}}
$$

where $\alpha$ is the thermal diffusivity, $\dot{\omega}_{\mathrm{F}}$ is the consumption rate of the fuel, and $\rho_{\mathrm{F}}$ is the initial density of the fuel in the mixture. The consumption rate of the fuel is evaluated at the flame temperature of the fuel since the high 
temperature kinetics in the reaction zone dominates. For an autoignition event, a linear relationship between the ratio of fuel density to fuel consumption rate and ignition delay is assumed:

$$
\frac{\rho_{\mathrm{F}}}{\dot{\omega}_{\mathrm{F}}\left(T_{\mathrm{f}}\right)}=\mathrm{C}_{\text {ign }} \tau_{\text {ign }}
$$

where $\mathrm{C}_{\text {ign }}$ is a proportionality constant.

Using the high activation energy asymptotics for a thermal explosion, given in Law [34], and comparing it to Eq. (24), the coefficient $\mathrm{C}_{\text {ign }}$ is deduced to be

$$
\mathrm{C}_{\text {ign }}=\gamma \beta \mathcal{A} Y_{\mathrm{F}} \exp \left(-\mathcal{A} \frac{\beta}{\beta+1}\right),
$$

where $\gamma$ is the heat capacity ratio, $\beta$ is the heat release parameter, and $Y_{\mathrm{F}}$ is the mass fraction of the fuel. The heat release parameter, $\beta=\Delta H_{\mathrm{C}} / c_{p} T \approx\left(T_{\mathrm{f}}-T\right) / T$, compares the heat of combustion, $\Delta H_{\mathrm{C}}$, to the initial enthalpy of the mixture, $c_{p} T$.

Equation (24) implicitly assumes that a thermal explosion is the mechanism for ignition. For dilute strong ignition events in RCMs, chain branching is more likely the impetus for ignition. However, since temperature is the primary inhomogeneity due to heat loss to the walls and radical concentrations are assumed to vary negligibly throughout the mixture, flame kernels likely result from thermal explosion. Hence, the scaling given in Eq. (24) is considered appropriate for this demarcation.

Using Eq. (22) and substituting the Zeldovich relation for the speed of the ignition front and the turbulent Damköhler number in the same manner as discussed in Sec. 2.2.1, the Sankaran criterion is found to be

$$
\operatorname{Da}_{\mathrm{t}}=\frac{\mathcal{T}_{T}^{2} \mathcal{A}}{5 \operatorname{Pr} \gamma \beta Y_{\mathrm{F}}} \exp \left(\mathcal{A} \frac{\beta}{\beta+1}\right) .
$$

Hence, the Sankaran criterion is independent to the Reynolds number. However, additional dependence on the chemical kinetics beyond that encapsulated by the turbulent Damköhler number appears in explicit form via the Arrhenius factor. This indicates a potential difficulty with the regime demarcation in the region of negative temperature coefficient (NTC) for a given fuel. As the Arrhenius factor becomes less in the NTC region, the critical Damköhler number for mild ignition correspondingly lessens and potentially becomes negative. A negative Damköhler limit implies that mild ignition could occur from cold-spots; however in these cases, non-ideal ignition is more likely to occur within the thermal boundary layer. Hence, this demarcation should be viewed as a conservative estimate of where fuels show evidence of non-ideal ignition. Additionally, a compositional dependence is shown by the fuel mass fraction; however, for narrow ranges of equivalence ratio for a given fuel, this factor is not expected to appreciably affect the demarcation. The boundary given by Eq. (26) is shown in Fig. 2 and separates mild and mixed regimes.

\subsubsection{Pre-ignition}

Radical build-up during the compression stroke constrains RCMs to long test times [1]. Hence, a straight-forward demarcation of the regime where radical build-up and pre-ignition appreciably affect the measurement of ignition delay is a comparison of the compression time to the ignition delay:

$$
\frac{\tau_{\text {comp }}}{\tau_{\text {ign }}}=1,
$$

where the compression time is related to the speed of piston by $\tau_{\text {comp }}=L / U_{\mathrm{P}}$, where $L$ is the stroke length. Substituting the relations for the turbulent Damköhler number, compression time, turbulent intensity, and $\mathrm{C}_{l}$, Eq. (27) can be rewritten as

$$
\mathrm{Da}_{\mathrm{t}}=\frac{\mathrm{C}_{l} d}{\mathcal{T}_{u} L} .
$$
272 pression ratio, CR:

$$
\mathrm{Da}_{\mathrm{t}}=\frac{\mathrm{C}_{l}}{\mathcal{T}_{u} \mathrm{AR}(\mathrm{CR}-1)},
$$

where the aspect ratio is the ratio of the length to the diameter of the test section, and the compression ratio is the ratio of the initial to the final volume of the test gas.

Considering the typical operational range of RCMs, this criterion is likely somewhat conservative. Also, much like heat transfer, the effect of radical build-up can be incorporated in modeling efforts to extend the operational range of facilities. However, the facility is ultimately limited by the occurrence of pre-ignition during the compression stroke. Additionally, note that the scaling relation given by Eq. 27 is essentially a simplification of the Livengood-Wu knock integral [35], where the ignition delay is taken to be invariant with the compression stroke. The boundary given by Eq. (29) is shown in Fig. 2 with a representative value of Dat $=10$. Damköhler numbers above this delineation indicate that radical build-up likely becomes a significant effect, and ultimately, where pre-ignition may occur during the compression stroke. 


\subsection{Heat transfer}

RCMs operate in a long ignition delay time regime, which is amenable to measuring low-temperature chemical kinetics. Hence, heat loss to the walls of an RCM can significantly affect the measured ignition delay time due to an appreciable decrease in pressure and temperature. Heat loss is taken into account in the majority of models of RCM experiments via the "adiabatic core hypothesis" as discussed in the Introduction. Heat loss is typically deduced a posteriori through a non-reactive pressure-trace and the isentropic relation

$$
\rho(t)=\rho_{0}\left[\frac{p(t)}{p_{0}}\right]^{\frac{1}{\gamma}},
$$

where the subscript "0" indicates the time at TDC to avoid confusion in this subsection. Integrating Eq. (1) over the adiabatic core and incorporating the isentropic relation, given by Eq. (30), the model equation for RCMs with heat loss is thus

$$
\rho c_{v} \frac{\mathrm{d} T}{\mathrm{~d} t}+\sum_{i=1}^{N_{\mathrm{s}}} e_{i} \dot{\omega}_{i}=\frac{1}{\gamma} \frac{\mathrm{d} p(t)}{\mathrm{d} t},
$$

where the term on the right-hand-side is interpreted as the isentropic pressure work done by the expansion of the adiabatic core.

\subsubsection{Thermal quenching}

A reactive mixture may not ignite in an RCM experiment due to heat loss. A reactive mixture's propensity for thermal quenching can be estimated by first assuming an exponential decay of temperature in a representative mixture:

$$
T=T_{0} \exp \left(-\frac{t}{\tau_{Q}}\right)
$$

where $\tau_{Q}$ is the heat loss time constant, which in general can be estimated from the initial slope at the end of the compression of a pressure-trace from an RCM experiment. Equation (32) may be derived from the transient heat equation using isothermal walls and truncating the resultant infinite series. Assuming simplified chemistry and the evolution of the temperature profile given by Eq. (32), Eq. (31) becomes

$$
\frac{\mathrm{d} T}{\mathrm{~d} t}=\frac{\Delta H_{\mathrm{C}}}{c_{v}} B Y_{\mathrm{F}} \exp \left(-\frac{E_{\mathrm{A}}}{\mathcal{R} T}\right)-\frac{T_{0}}{\tau_{Q}} \exp \left(-\frac{t}{\tau_{Q}}\right) .
$$

Since the heat loss term is monotonically decreasing with temperature and the chemical source term is a decreasing function of temperature, it can be deduced that ignition is guaranteed to occur when the initial slope of the temperature derivative is zero. Using this criterion and evaluating Eq. (33) at the initial time, we have the following condition:

$$
0=\frac{\Delta H_{\mathrm{C}}}{c_{v}} B Y_{\mathrm{F}} \exp \left(-\frac{E_{\mathrm{A}}}{\mathcal{R} T_{0}}\right)-\frac{T_{0}}{\tau_{Q}} .
$$

Noting that $\rho B Y_{\mathrm{F}} \exp \left(-E_{\mathrm{A}} / \mathcal{R} T_{0}\right)=\dot{\omega}_{\mathrm{F}}$, applying Eq. (24), and rearranging, Eq. (34) becomes:

$$
\frac{\tau_{Q}}{\tau_{\text {ign }}}=\mathcal{A}
$$

Assuming the relationship $\tau_{Q}=b^{2} / \alpha$, where $b$ is the characteristic heat transfer length scale (taken to be the volume of the test section divided by its surface area), and applying the definition of the turbulent Reynolds number and the Prandtl number to Eq. (35), the thermal quenching limit is found to be:

$$
\mathrm{Da}_{\mathrm{t}}=\left(\mathrm{C}_{l} \mathrm{C}_{Q}\right)^{2} \mathcal{A}\left(\operatorname{Pr} \mathrm{Re}_{\mathrm{t}}\right)^{-1}
$$

where $\mathrm{C}_{Q}$ is a constant that relates the characteristic heat transfer length scale to the diameter of the $\mathrm{RCM}: d=\mathrm{C}_{Q} b$. For example, $\mathrm{C}_{Q}$ for a right circular cylinder with a height equal to its diameter would be $1 / 6$. The line given by Eq. (36) segregates the quenched and non-adiabatic regimes in Fig. 2 and has a slope of -1.

\subsubsection{Loss of adiabaticity}

A delineation of the regime where heat transfer effects become appreciable can be derived by examining the effect that the change in temperature has on the ignition delay. Assuming an Arrhenius form for the ignition delay, the following relation holds for small changes in temperature during the ignition process due to heat loss

$$
\frac{\Delta \tau_{\text {ign }}}{\tau_{\text {ign }, 0}}=-\mathcal{A} \frac{\Delta T}{T_{0}}
$$


where $\Delta \tau_{\text {ign }}$ denotes the change in the ignition delay due to a change in temperature $\Delta T$. Using the temporal change in temperature during an ignition process, given by Eq. (32), to estimate the characteristic ignition temperature with heat loss, the change in temperature is given by

$$
\Delta T=T_{0} \exp \left(-\frac{\tau_{\text {ign }}}{\tau_{Q}}\right)-T_{0} .
$$

Substituting Eq. (38) into Eq. (37), the following is obtained:

$$
\frac{\Delta \tau_{\text {ign }}}{\tau_{\text {ign }, 0}}=-\mathcal{A}\left[\exp \left(-\frac{\tau_{\text {ign }}}{\tau_{Q}}\right)-1\right] .
$$

321 Realizing that the heat loss time scale must be much larger than the ignition delay in cases where heat loss is negligible, the exponential term in Eq. (37) is approximated by the first two terms in the Taylor expansion giving

$$
\frac{\Delta \tau_{\text {ign }}}{\tau_{\text {ign }, 0}}=\mathcal{A} \frac{\tau_{\text {ign }}}{\tau_{Q}}
$$

Using the same scaling as before for the heat transfer time scale, the demarcation for significant heat loss in RCM experiments is given by

$$
\mathrm{Da}_{\mathrm{t}}=\frac{\left(\mathrm{C}_{l} \mathrm{C}_{Q}\right)^{2}}{\epsilon} \mathcal{A}\left(\operatorname{Pr}_{\mathrm{Re}}\right)^{-1},
$$

where $\epsilon=\Delta \tau_{\text {ign }} / \tau_{\text {ign }}$, and is necessarily small for the aforementioned assumptions to hold. Hence, the division between the adiabatic and non-adiabatic regimes shows the same scaling as the thermal quenching demarcation. However, the former requires much larger Damköhler numbers than the latter since $\epsilon \ll 1$. Equation (41) separates the adiabatic and non-adiabatic regimes in Fig. 2, and has a slope of -1 .

\section{Discussion}

A summary of the scaling relations is compiled in Table 1. This table confirms that the primary non-dimensional groups affecting the turbulent Damköhler number are the turbulent Reynolds number and the Arrhenius factor.

An interpretation of the turbulent Reynolds number is a ratio of viscous to turbulent time scales as shown in Eq. (5). Since the turbulent Damköhler number is proportional to the turbulent time scale, a slope of -1 in the regime diagram indicates an independence to turbulence of the demarcation. Hence, the turbulent Reynolds number shows substantial influence in determining the ignition character delineations.

\subsection{Influence of the Arrhenius factor}

The Arrhenius factor influences all of the demarcations besides the boundary for the quiescent regime. Assuming simplified chemistry, the logarithmic sensitivity of the species production rate to temperature is given by

$$
\frac{\partial \ln \dot{\omega}_{\mathrm{F}}}{\partial \ln T}=\mathcal{A}
$$

Hence, the Arrhenius factor is a parameter that indicates the sensitivity of the reaction chemistry to a perturbation in temperature. As discussed in the Introduction, the effect of the sensitivity of a mixture was studied by Meyer and Oppenheim [11] where it was found that strong-to-mild transition was delineated by an isopleth of ignition delay sensitivity. This criterion is given by

$$
\frac{\partial \tau_{\text {ign }}}{\partial T}=\mathrm{C}_{\mathrm{S}}
$$

where $\mathrm{C}_{\mathrm{S}}$ is determined from experiment for a given mixture. Rewriting the Sankaran criterion, given in Eq. (22), and applying Zeldovich's relation for the speed of the reaction front, the following relation results:

$$
\frac{\partial \tau_{\text {ign }}}{\partial T}=\left(\frac{\partial T}{\partial \xi} S_{\mathrm{L}}\right)^{-1}
$$

The qualitative similarity between Eqs. (43) and (44) is apparent. However, the two are distinct since the laminar flame speed of a mixture varies significantly with temperature and pressure. Mansfield et al. assumed a constant temperature gradient within RCMs; however, using the analysis outlined in Sec. 2.2.1, the temperature gradient is found to have the following thermodynamic scaling:

$$
\frac{\partial T}{\partial \xi} \sim\left(p T^{2-g}\right)^{1 / 2}
$$

where the parameter $g$ accounts for the scaling of the kinematic viscosity with temperature, which is typically between 1.5-2. Hence, the temperature gradient shows significant dependence on the thermodynamic conditions at top dead center in addition to the piston speed and the design of the RCM. 


\subsection{Comparison of the regime diagram to experiments}

A comparison of the ignition quality demarcations derived in Sec. 2.2 is presented in Fig. 4. All ignition delay time data except for the University of Michigan syngas data were taken directly from experiments. For this data, adiabatic, isochoric reactors using the $\mathrm{Li}$ et al. [36] $\mathrm{C}_{1}$-mechanism at the specified thermodynamic conditions were used since significant deviation in the experimental ignition delay time from the reactor model is found for the mixed/DDT and the mild ignition cases. Additionally, the turbulent Reynolds and Damköhler numbers were computed using the relations specified in Sec. 2.1.3. The parameters $\mathcal{T}_{u}$ and $\mathrm{C}_{l}$ were taken to be $2 \%$ and $10 \%$, respectively. Table 2 gives the machine-dependent parameters used to create Fig. 4.

Considering the highly stochastic nature of inhomogeneous ignition events, the regime diagram shows favorable agreement with the experimental data. The transition between the regimes is quite evident in the syngas data. However, the iso-octane data shows robustness in the mixed ignition regime; this is expected since two-stage ignition phenomena tend to homogenize the mixture, which decreases the propensity towards mixed or mild ignition [37]. Additionally, fuels such as iso-octane exhibit a decreasing Arrhenius factor as it approaches the NTC region; hence, the static demarcations with respect to the Arrhenius factor become increasingly conservative. Additionally, the n-pentane experiments in [38] were also conducted within the NTC region. The character of the ignition event is not reported for the NUI Galway and Case Western Reserve machines, but both are within the regimes where strong or mixed ignition is likely.

\subsection{Mild ignition demarcation}

The demarcation of mild ignition can be interpreted as the competition between heat dissipation and heat addition due to the reaction. For an ignition kernel, the competition between heat dissipation and chemistry is given by

$$
\frac{\text { Dissipation }}{\text { Chemistry }}=\frac{\rho c_{p} \alpha \frac{\mathrm{d}^{2} T}{\mathrm{~d} \xi^{2}}}{\Delta H_{\mathrm{C}} \dot{\omega}_{\mathrm{F}}}=1,
$$

where it can be seen that values of less than one lead to thermal ignition. Assuming symmetry at the center of the ignition kernel, $\mathrm{d}^{2} T / \mathrm{d} \xi^{2} \approx 2 T^{\prime} / \lambda_{\mathrm{T}}^{2}$ for small kernel sizes. Rearranging Eq. (46) and expressing in terms of a turbulent Damköhler number, the following is obtained:

$$
\mathrm{Da}_{\mathrm{t}}=\frac{\gamma \mathcal{T}_{T} \mathcal{A}}{5 \operatorname{Pr}}
$$

Hence, Eq. (47) shows that kernel formation is independent to the turbulent Reynolds number as in the Sankaran criterion. Additionally, Eq. (47) indicates that turbulence within the RCM has an ameliorating effect, reducing the propensity towards kernel formation. However, this demarcation would be present for turbulent Damköhler numbers low enough that heat transfer effects would likely dominate in the operational ranges of RCMs.

\subsection{Adiabatic core hypothesis}

The adiabatic core hypothesis is presumed to be valid in the modeling of heat transfer effects in RCMs. The adiabatic core hypothesis relies on the supposed isentropic expansion of the test gas within an RCM. However, it is shown that diffusive losses will cause this hypothesis to break down at long test times. Hence, there should be a critical thermal boundary layer thickness, $\delta$, in relation to the characteristic heat transfer dimension, $b$, such that this hypothesis is valid. Again, $b$ is taken to be the combustion chamber's ratio of volume to surface area. For this critical value of the boundary layer thickness, the relationship to the ignition delay time is

$$
\delta=\delta_{0}+\sqrt{\alpha \tau_{\text {ign }}}
$$

where $\delta_{0}$ is the size of the thermal boundary layer at TDC. Dividing through by $b$, squaring, and substituting the definitions of the turbulent Damköhler and Reynolds numbers, the criterion becomes

$$
\mathrm{Da}_{\mathrm{t}}=\left(\frac{\delta-\delta_{0}}{b}\right)^{-2}\left(\mathrm{C}_{Q} \mathrm{C}_{l}\right)^{2}\left(\operatorname{Pr}_{\mathrm{Re}}\right)^{-1}
$$

where the ratio, $\left(\delta-\delta_{0}\right) / b$, is determined from experiments or more advanced heat-transfer models. Comparing this to the thermal quenching criterion given by Eq. (36), it appears that the invalidation of the adiabatic core hypothesis is unlikely to be a significant effect since mixtures without an NTC region will likely quench before this effect is appreciable. Therefore, this demarcation is not included in the representative comparison depicted in Fig. 2 since it is primarily designed for syngas mixtures. However, for mixtures within the NTC region, the adiabatic core hypothesis may break down before thermal quenching due to the decreased Arrhenius factor. 


\subsection{The operational range of a rapid compression machine}

A comparison of the operational range of a representative RCM with a lean iso-octane mixture at several pressures is depicted in Fig. 5. The test duration is shown to be limited by the mild-ignition demarcation given by the Sankaran criterion and the prevalence of radical build-up during the compression stroke. The Sankaran criterion may be computed precisely using Eq. (22), substituting the Zeldovich relation for the speed of the ignition front, and estimating the temperature stratification; however for simplicity, a critical turbulent Damköhler number, Dat,crit, of 0.3 is used since this value corresponded well to the experimental data given in Fig. 4. Additionally, the radical buildup limit illustrated in Fig. 5 may be computed more precisely using a realistic piston speed profile in conjunction with a variable volume reactor model, and estimating the error incurred in the ignition delay measurement by neglecting the compression stroke.

Within this operational range, heat transfer effects are expected to be significant, and the upper limit of the test times may approach the regime where the break down of the adiabatic core hypothesis and thermal quenching could occur. Also, the ejection of corner vortices into the core fluid may be present as well if the crevice design is not adequate.

From Fig. 5 it is evident that the operational range of a rapid compression machine is dependent on the operating conditions of the machine; there is an implicit trade-off with respect to the piston speed; higher piston speeds allow for an increased operational range at higher temperatures due to the reduction of the compression time; however, higher piston speeds can increase the gas dynamic fluctuations in the test gas [7, 8], which can lead to non-ideal ignition at lower temperatures. Hence, an effective test strategy could be to tailor the compression stroke with respect to the desired test-time to increase the operational range of the RCM.

\section{Conclusions}

Rapid compression machines allow for low- and intermediate-temperature chemical kinetic studies in thermodynamic regimes under fairly ideal conditions. However, heat transfer and turbulence can significantly affect the character of the ignition process and obfuscate the interpretation of chemical kinetic studies. By considering the competition between turbulent, chemical, and heat transfer effects, a regime diagram was developed to guide RCM experiments. The demarcations of the strong, mixed/DDT, and mild ignition regimes were built upon work on DDT and mild ignition and translated into canonical Damköhler number and Reynolds number coordinates. The Damköhler and Reynolds number scalings were found to be particularly well-suited to describe ignition phenomena in RCMs because of their incorporation of the competing chemical, mixing, and dissipation time scales. Additionally, delineations of the heat transfer regimes were made by applying the prevalent "adiabatic core hypothesis," and the Arrhenius form for the ignition delay.

The regime diagram was compared against experiments, involving different RCM geometries, operating conditions, and fuel mixtures, and good agreement was found, indicating that the combustion regime diagram presented in this paper could be used as a practical guide to ignition phenomena within RCMs.

\section{Acknowledgments}

The authors acknowledge financial support through the Air Force Office of Scientific Research under Award No. FA9550-14-1-0219 with Dr. Chiping Li as program manager. Additionally, the authors acknowledge support through the US DOE Vehicle Technologies Program with Gurpreet Singh and Leo Breton as program managers. Argonne National Laboratory is operated by UChicago Argonne, LLC under Contract No. DE-AC02-06CH11357. The US Government retains for itself, and others acting on its behalf, a paid-up non-exclusive, irrevocable worldwide license in said article to reproduce, prepare derivative works, distribute copies to the public, and perform publicly, by or on behalf of the Government. An early version of this regime diagram was presented at the Second International RCM Workshop in Berkeley [39].

\section{References}

[1] C. J. Sung, H. J. Curran, Prog. Energy Combust. Sci. 44 (2014) 1-18.

[2] R. K. Hanson, D. F. Davidson, Prog. Energy Combust. Sci. 44 (2014) 103-114.

[3] F. L. Dryer, F. M. Haas, J. Santner, T. Farouk, M. Chaos, Prog. Energy Combust. Sci. 44 (2014) $19-39$.

[4] P. Park, J. C. Keck, SAE Paper 900027 (1990).

[5] D. Lee, S. Hochgreb, Combust. Flame 114 (1998) 531-545.

[6] G. Mittal, C.-J. Sung, Combust. Flame 145 (2006) 160-180.

[7] M. Ihme, Combust. Flame 159 (2012) 1592-1604.

[8] P. Hamlington, M. Ihme, Flow, Turb. Combust. 93 (2014) 93-124.

[9] P. Guibert, A. Keromnes, G. Legros, Flow, Turb. Combust. 84 (2010) 79-95.

[10] J. C. Livengood, W. A. Leary, Ind. Eng. Chem. 43 (1951) 2797-2805.

[11] J. W. Meyer, A. K. Oppenheim, Proc. Combust. Inst. 13 (1971) 1153-1164.

[12] D. J. Vermeer, J. W. Meyer, A. K. Oppenheim, Combust. Flame 18 (1972) 327-336.

[13] K. Fieweger, R. Blumenthal, G. Adomeit, Proc. Combust. Inst. 25 (1994) 1579-1585. 
451 [14] K. Fieweger, R. Blumenthal, G. Adomeit, Combust. Flame 109 (1997) 599-619.

452 [15] H. Yamashita, J. Kasahara, Y. Sugiyama, A. Matsuo, Combust. Flame 159 (2012) $2954-2966$.

453 [16] Y. Uygun, S. Ishihara, H. Olivier, Combust. Flame 161 (2014) 2519-2530.

454 [17] S. M. Walton, X. He, B. T. Zigler, M. S. Wooldridge, A. Atreya, Combust. Flame 150 (2007) $246-262$.

455 [18] S. M. Walton, X. He, B. T. Zigler, M. S. Wooldridge, Proc. Combust. Inst. 31 (2007) $3147-3154$.

456 [19] A. Mansfield, M. S. Wooldridge, Combust. Flame 161 (2014) 2242-2251.

457 [20] A. Mansfield, M. S. Wooldridge, H. Di, X. He, Fuel 139 (2015) 79-86.

458 21] C. Strozzi, A. Mura, J. Sotton, M. Bellenoue, Combust. Flame 159 (2012) 3323-3341.

459 [22] H. Wu, M. Ihme, Combust. Flame 161 (2014) 2317-2326.

$460[23]$ N. Peters, B. Kerschgens, G. Paczko, SAE Paper 2013-01-1109 (2013).

461 [24] S. B. Pope, Turbulent Flows, Cambridge University Press, Cambridge, 2000.

462 [25] Y. B. Zeldovich, V. B. Librovich, G. M. Makhviladze, G. I. Sivashinsky, Acta Astronautica 15 (1970) $313-321$

463 [26] J. H. Lee, R. Knystautas, N. Yoshikawa, Acta Astronautica 5 (1978) 971-982.

464 [27] J. H. Lee, The Detonation Phenomenon, Cambridge University Press, Cambridge, 2008.

465 [28] Y. B. Zeldovich, Combust. Flame 39 (1980) 211-214.

466 [29] X. Gu, D. Emerson, D. Bradley, Combust. Flame 133 (2003) 63-74.

467 [30] K. Grogan, M. Ihme, Proc. Combust. Inst. 35 (2014) 2181-2189.

468 [31] R. Blumenthal, K. Fieweger, K. H. Komp, G. Adomeit, Combust. Sci. Tech. 113 (1996) $137-166$.

469 [32] R. Sankaran, H. G. Im, E. R. Hawkes, J. H. Chen, Proc. Combust. Inst. 30 (2005) 875-882.

470 [33] S. R. Turns, An Introduction to Combustion: Concepts and Applications, McGraw Hill, 2000.

471 [34] C. K. Law, Combustion Physics, Cambridge University Press, Cambridge, 2006.

472 [35] J. C. Livengood, P. Wu, Symp. Int. Combust. Proc. 5 (1955) 347-356.

473 [36] J. Li, Z. Zhao, A. Kazakov, M. Chaos, F. L. Dryer, J. Scire, Jr., Int. J. Chem. Kinet. 39 (2007) $109-136$.

474 [37] J. F. Griffiths, B. J. Whitaker, Combust. Flame 131 (2002) 386-399.

475 [38] B. Marks, O. Mathieu, R. Archuleta, E. L. Petersen, J. Bugler, A. Heufer, H. Curran, AIAA Paper 2013-0160 (2013).

476 [39] International RCM Workshop, Available: http://www.transportation.anl.gov/rcmworkshop/, August 1, 2014. Berkeley, CA.

477 [40] M. T. Donovan, X. He, B. T. Zigler, T. R. Palmer, M. S. Wooldridge, A. Atreya, Combust. Flame 137 (2004) $351-365$.

478 [41] H. Di, X. He, P. Zhang, Z. Wang, M. S. Wooldridge, C. K. Law, C. Wang, S. Shuai, J. Wang, Combust. Flame 161 (2014) 2531-2538.

479 [42] G. Mittal, A Rapid Compression Machine - Design, Characterization, and Autoignition Investigation, Ph.D. thesis, Case Western 480 Reserve University, 2006.

481 [43] W. Affleck, A. Thomas, Proc. Inst. Mech. Eng. 183 (1968) 365-387.

482 [44] P. Pepiot-Desjardins, H. Pitsch, Combust. Flame 154 (2008) 67-81. 


\begin{tabular}{|c|c|c|c|c|c|}
\hline Demarcation & $a$ & $b$ & $c$ & $d$ & $e$ \\
\hline Quiescent/Turbulent & $\infty,-3.3$ & 0 & 0 & 0 & 0 \\
Pre-Ignition/Strong & 0 & 0 & 0 & 0 & 0 \\
Strong/Mixed & $1 / 2$ & 1 & 0 & 0 & -1 \\
Mixed/Mild & 0 & 1 & -1 & 1 & 0 \\
Ignition/Quenching & -1 & 1 & -1 & 0 & 0 \\
Adiabatic/Non-Adiabatic & -1 & 1 & -1 & 0 & 0 \\
\hline
\end{tabular}

Table 1: Summary of the scaling relations found for the non-dimensional groups for the regime demarcation. Damköhler scaling has the form $\mathrm{Da}_{\mathrm{t}} \sim \operatorname{Re}_{\mathrm{t}}^{a} \mathcal{A}^{b} \operatorname{Pr}^{c} \beta^{d} \mathrm{M}^{e}$. 


\begin{tabular}{|c|c|c|c|c|c|}
\hline Machine & $d[\mathrm{~cm}]$ & $U_{\mathrm{P}}[\mathrm{m} / \mathrm{s}]$ & $\mathcal{T}_{u}^{*}[\%]$ & $\mathrm{C}_{l}^{*}[\%]$ & $\tau_{\mathrm{t}}[\mathrm{ms}]$ \\
\hline Michigan [40] & 5.08 & 30.0 & 2 & 10 & 8.47 \\
Tsinghua [41] & 5.08 & 16.7 & 2 & 10 & 15.2 \\
Case Western [42] & 5.08 & 6.64 & 2 & 10 & 38.2 \\
NUI Galway [43, 38] & 3.81 & 10.1 & 2 & 10 & 18.8 \\
\hline
\end{tabular}

Table 2: Summary of the relevant machine-dependent parameters used to create Fig. 4. The turbulent time scale was estimated using Eq. (12). The piston velocity was either taken directly or calculated by dividing the stroke length of the RCM by the compression time. Quantities with an $(*)$ were estimated. 


\section{List of Figures}

1 (Color online) Typical operational boundaries of shock-tubes, rapid compression machines, and flow reactors. A comparison to a representative ignition delay curve of iso-octane is included; ignition delay of iso-octane obtained from the reduced mechanism of Pepiot-Desjardins and Pitsch [44] at an equivalence ratio of 0.6 and a pressure of $20 \mathrm{bar} . \ldots \ldots \ldots \ldots \ldots \ldots \ldots$

2 (Color online) Diagram illustrating the combustion regimes occurring in RCM experiments. The abscissa is the turbulent Reynolds number given by Eq. (4) and the ordinate is the turbulent Damköhler number given by Eq. (3). The BLACK lines indicates demarcations of the quality of ignition, and the RED lines show demarcations relevant to heat transfer. The GRAY area for the mixed/DDT region is meant to emphasize that this is a transitional regime. . . . . . . . . . . .

3 (Color online) Schematic of the turbulent field within a rapid compression machine at the time at the end of compression. $l$ is the integral length scale, $\lambda_{\mathrm{T}}$ is the Taylor microscale, $\eta$ is the Kolmogorov scale, $d$ is the diameter of the RCM, $\delta$ is the length of the thermal boundary layer, and $\xi$ is the characteristic length scale of an ignition kernel. . . . . . . . . . . . . . . . . . . 19

4 (Color online) Comparison of the demarcations of ignition quality to experiment. BLACK symbols indicate strong ignition, RED symbols indicate mixed/DDT ignition, BLUE symbols indicate mild ignition, and GREEN symbols indicate that the quality of ignition was not reported. $\square$ taken from Mansfield et al. [19], o taken from Mansfield et al. [20], $\times$ taken from Mittal [42], and $\triangle$ taken from Marks et al. [38]. The sensitivity of each point to a factor of two increase in the assumed parameters

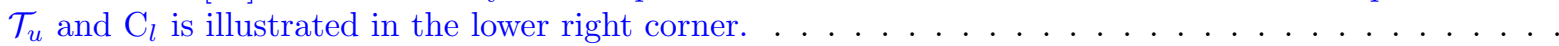

5 (Color online) Operational range of a representative RCM with respect to the mild ignition and radical build-up limits. The GRAY area highlights the operational limits for a machine with a compression time of $\tau_{\text {comp }}=10 \mathrm{~ms}$ and a turbulence time scale of $\tau_{\mathrm{t}}=20 \mathrm{~ms}$. The critical Damköhler number for the onset of mild ignition, $\mathrm{Da}_{\mathrm{t}, \mathrm{crit}}$, is taken to be 0.3 . The ignition delay curves of iso-octane are obtained from the reduced mechanism of Pepiot-Desjardins and Pitsch [44] at an equivalence ratio of

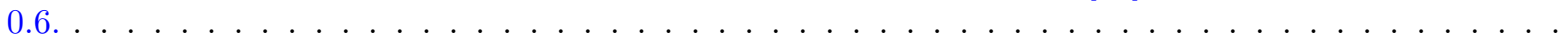




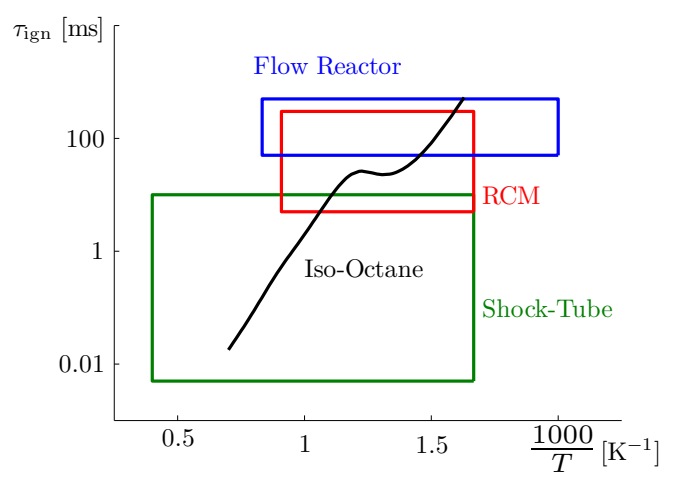

Figure 1: (Color online) Typical operational boundaries of shock-tubes, rapid compression machines, and flow reactors. A comparison to a representative ignition delay curve of iso-octane is included; ignition delay of iso-octane obtained from the reduced mechanism of Pepiot-Desjardins and Pitsch [44] at an equivalence ratio of 0.6 and a pressure of 20 bar. 


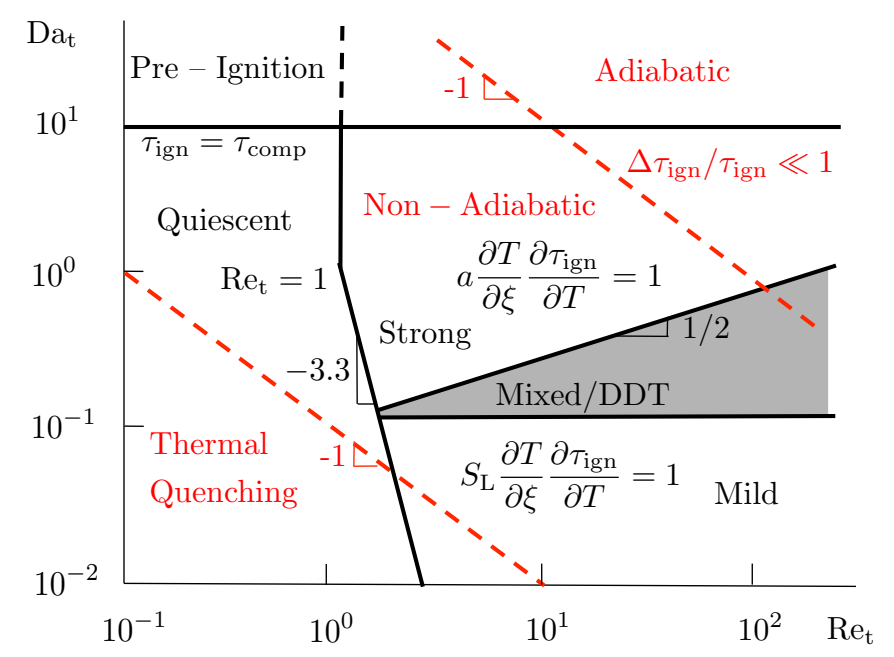

Figure 2: (Color online) Diagram illustrating the combustion regimes occurring in RCM experiments. The abscissa is the turbulent Reynolds number given by Eq. (4) and the ordinate is the turbulent Damköhler number given by Eq. (3). The BLACK lines indicates demarcations of the quality of ignition, and the RED lines show demarcations relevant to heat transfer. The GRAY area for the mixed/DDT region is meant to emphasize that this is a transitional regime. 


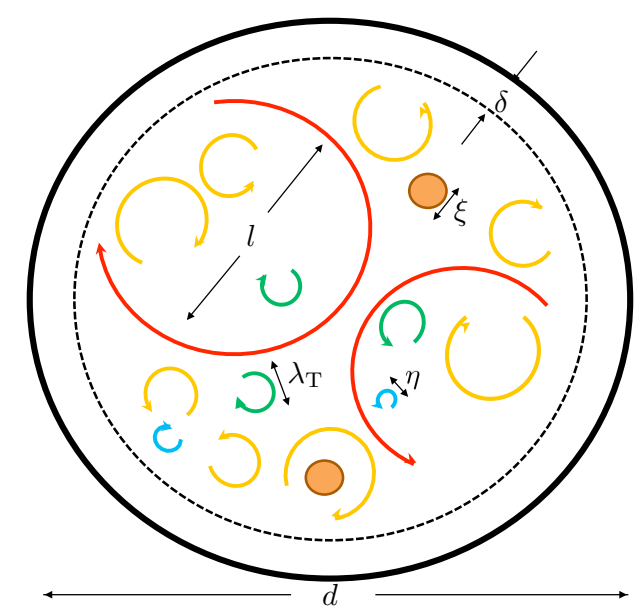

Figure 3: (Color online) Schematic of the turbulent field within a rapid compression machine at the time at the end of compression. $l$ is the integral length scale, $\lambda_{\mathrm{T}}$ is the Taylor microscale, $\eta$ is the Kolmogorov scale, $d$ is the diameter of the RCM, $\delta$ is the length of the thermal boundary layer, and $\xi$ is the characteristic length scale of an ignition kernel. 


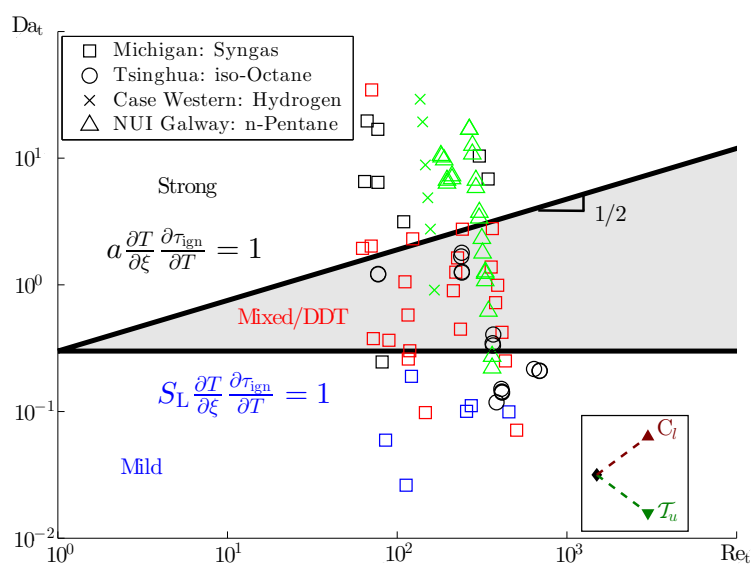

Figure 4: (Color online) Comparison of the demarcations of ignition quality to experiment. BLACK symbols indicate strong ignition, RED symbols indicate mixed/DDT ignition, BLUE symbols indicate mild ignition, and GREEN symbols indicate that the quality of ignition was not reported. $\square$ taken from Mansfield et al. [19], ○ taken from Mansfield et al. [20], $\times$ taken from Mittal [42], and $\triangle$ taken from Marks et al. [38]. The sensitivity of each point to a factor of two increase in the assumed parameters $\mathcal{T}_{u}$ and $\mathrm{C}_{l}$ is illustrated in the lower right corner. 


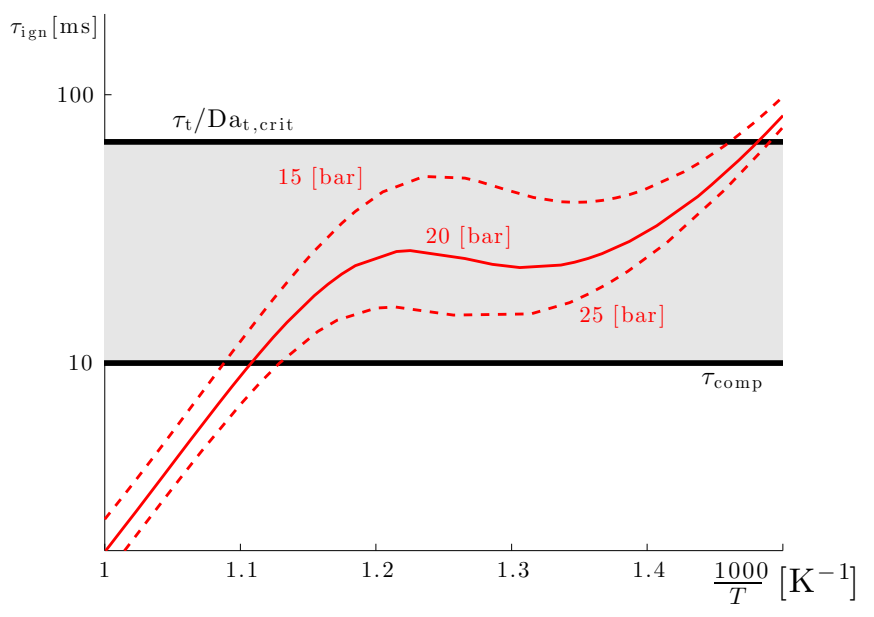

Figure 5: (Color online) Operational range of a representative RCM with respect to the mild ignition and radical build-up limits. The GRAY area highlights the operational limits for a machine with a compression time of $\tau_{\text {comp }}=10 \mathrm{~ms}$ and a turbulence time scale of $\tau_{\mathrm{t}}=20 \mathrm{~ms}$. The critical Damköhler number for the onset of mild ignition, Dat,crit, is taken to be 0.3. The ignition delay curves of iso-octane are obtained from the reduced mechanism of Pepiot-Desjardins and Pitsch [44] at an equivalence ratio of 0.6. 